\title{
Iterative approximation of fixed points of quasi-contraction mappings in cone metric spaces
}

\author{
Petko D Proinov* and Ivanka A Nikolova
}

\section{"Correspondence:}

proinov@uni-plovdiv.bg

Faculty of Mathematics and

Informatics, University of Plovdiv,

Plovdiv, 4000, Bulgaria

\begin{abstract}
In this paper, we establish a full statement of Ćirić's fixed point theorem in the setting of cone metric spaces. More exactly, we obtain a priori and a posteriori error estimates for approximating fixed points of quasi-contractions in a cone metric space. Our result complements recent results of Zhang (Comput. Math. Appl. 62:1627-1633, 2011), Ding et al. (J. Comput. Anal. Appl. 15:463-470, 2013) and others.

MSC: Primary 54H25; secondary 47H10; 46A19
\end{abstract}

Keywords: Picard iteration; cone metric space; solid vector space; fixed points; quasi-contractions; error estimates

\section{Introduction}

In this paper, we study fixed points of quasi-contraction mappings in a cone metric space $(X, d)$ over a solid vector space $(Y, \preceq)$. Cone metric spaces have a long history (see Collatz [1], Zabrejko [2], Janković et al. [3], Proinov [4] and references therein). A unified theory of cone metric spaces over a solid vector space was developed in a recent paper of Proinov [4]. Recall that an ordered vector space with convergence structure $(Y, \preceq)$ is called:

- a solid vector space if it can be endowed with a strict vector ordering $(\prec)$;

- a normal vector space if the convergence of $Y$ has the sandwich property.

Every metric space $(X, d)$ is a cone metric space over $\mathbb{R}$ (with usual ordering and usual convergence). On the other hand, every cone metric space over a solid vector space is a metrizable topological space (see Proinov [4] and references therein). It is well known that a lot of fixed point results in cone metric setting can be directly obtained from their metric versions (see Du [5], Amini-Harandi and Fakhar [6], Feng and Mao [7], Kadelburg et al. [8], Asadi et al. [9], Proinov [4], and Ercan [10]).

For instance, for this purpose we can use the following theorem. This theorem shows that every cone metric is equivalent to a metric which preserves the completeness as well as some inequalities.

Theorem 1.1 ([4, Theorem 9.3]) Let $(X, d)$ be a cone metric space over a solid vector space $(Y, \preceq)$. Then there exists a metric $\rho$ on $X$ such that the following statements hold true.

(i) The topology of $(X, d)$ coincides with the topology of $(X, \rho)$. 
(ii) $(X, d)$ is complete if and only if $(X, \rho)$ is complete.

(iii) For $x, x_{1}, \ldots, x_{n} \in X, y, y_{1}, \ldots, y_{n} \in X$ and $\lambda_{1}, \ldots, \lambda_{n} \in \mathbb{R}$,

$$
d(x, y) \preceq \sum_{i=1}^{n} \lambda_{i} d\left(x_{i}, y_{i}\right) \quad \text { implies } \quad \rho(x, y) \leq \sum_{i=1}^{n} \lambda_{i} \rho\left(x_{i}, y_{i}\right) .
$$

In 1922, Banach [11] proved his famous fixed point theorem for contraction mappings. Banach's contraction principle is one of the most useful theorems in the fixed point theory. It has two versions: a short version and a full version. In a metric space setting its full statement can be seen, for example, in the monograph of Berinde [12, Theorem 2.1]. Recently, full statements of Banach's fixed point theorem in a cone metric spaces over a solid vector space were given by Radenović and Kadelburg [13, Theorem 3.3] and Proinov [4, Theorem 11.1].

Definition 1.2 ([14]) Let $(X, d)$ be a metric space. A mapping $T: X \rightarrow X$ is called a quasicontraction (with contraction constant $\lambda$ ) if there exists $\lambda \in[0,1)$ such that

$$
d(T x, T y) \leq \lambda \max \{d(x, y), d(x, T x), d(y, T y), d(x, T y), d(y, T x)\}
$$

for all $x, y \in X$.

There are a large number of generalizations of Banach's contraction principle (see, for example, [14-18] and references therein). In 1974, Ćirić [14] introduced contraction mappings and proved the following well known generalization of Banach's fixed point theorem.

Theorem 1.3 Let $(X, d)$ be a complete metric space and $T: X \rightarrow X$ be a quasi-contraction with contraction constant $\lambda$. Then the following statements hold true:

(i) Existence And uniQueness. $T$ has a unique fixed point $\xi$ in $X$.

(ii) Convergence of Picard iteration. For every starting point $x \in X$ the Picard iteration sequence $\left(T^{n} x\right)$ converges to $\xi$.

(iii) A PRIORI ERror estimate. For every point $x \in X$ the following a priori error estimate holds:

$$
d\left(T^{n} x, \xi\right) \leq \frac{\lambda^{n}}{1-\lambda} d(x, T x) \text { for all } n \geq 0 .
$$

Following Zhang [19], in the next definition, we define a useful binary relation between an ordered vector space $Y$ and the set of all subsets of $Y$. It plays a very important role in this paper as it is used to prove our main result.

Definition 1.4 ([19]) Let $(Y, \preceq)$ be an ordered vector space, $x \in Y$ and $A \subset Y$. We say that $x \preceq A$ if there exists at least one vector $y \in A$ such that $x \preceq y$.

In 2009, Ilić and Rakočević [20] generalized the concept of quasi-contraction to cone metric space as follows: A selfmapping $T$ of a cone metric space $(X, d)$ over an ordered vector space $(Y, \preceq)$ is called a quasi-contraction on $X$ if there exists $\lambda \in[0,1)$ such that

$$
d(T x, T y) \preceq \lambda\{d(x, y), d(x, T x), d(y, T y), d(x, T y), d(y, T x)\}
$$


for all $x, y \in X$. They proved the following result [20, Theorem 2.1]: Let $(X, d)$ be a cone metric space over a normal solid Banach space $(Y, \preceq)$; then every quasi-contraction $T$ of the type (3) has a unique fixed point in $X$, and for all $x \in X$ the Picard iterative sequence $\left(T^{n} x\right)$ converges to this fixed point. Kadelburg et al. [21, Theorem 2.2] improved this result by omitting the assumption of normality provided that $\lambda \in[0,1 / 2)$. Gajić and Rakočević [22, Theorem 3] proved this result for any contraction constant $\lambda \in[0,1)$. Rezapour et al. [23, Theorem 2.1] proved this result in the case when $Y$ is a solid topological vector space and $\lambda \in[0,1)$. Furthermore, Kadelburg et al. [8, Theorem 3.5(b)] proved that this result is equivalent to the short version of Ćirić's fixed point theorem.

In 2011, Zhang [19] presented the following new definition for quasi-contractions in cone metric spaces.

Definition 1.5 ([19]) Let $(X, d)$ be a cone metric space over an ordered vector space $(Y, \preceq)$. A mapping $T: X \rightarrow X$ is called a quasi-contraction (with contraction constant $\lambda$ ) if there exists $\lambda \in[0,1)$ such that

$$
d(T x, T y) \preceq \lambda \operatorname{co}\{d(x, y), d(x, T x), d(y, T y), d(x, T y), d(y, T x)\}
$$

for all $x, y \in X$.

By applying Theorem 1.1 to the first two conclusions of Theorem 1.3, we obtain the following fixed point theorem in a cone metric setting.

Theorem 1.6 Let $(X, d)$ be a complete cone metric space over a solid vector space $(Y, \preceq)$ and $T: X \rightarrow X$ be a quasi-contraction. Then the following statements hold true:

(i) EXISTENCE AND UNIQUeNess. $T$ has a unique fixed point $\xi$ in $X$.

(ii) Convergence of Picard iteration. For every starting point $x \in X$ the Picard iteration sequence $\left(T^{n} x\right)$ converges to $\xi$.

In 2011, Zhang [19] proved Theorem 1.6 in the case when $Y$ is a normal solid Banach space. In 2013, Ding et al. [24] proved this theorem in the case when $Y$ is a solid topological vector space.

In this paper, we establish a full statement of Ćirić's fixed point theorem in the setting of cone metric spaces. Our result complements Theorem 1.6. Thus it extends and complements the corresponding results of Zhang [19], Ding et al. [24], and others.

For some recent results on the topic, we refer the reader to [25-38]. In the papers [37, 38] one can find some applications of cone metric spaces to iterative methods for finding all zeros of polynomial simultaneously.

\section{Preliminaries}

In this section, we introduce some basic definitions and theorems of cone metric spaces over a solid vector space.

Definition 2.1 ([4]) Let $Y$ be a real vector space and $S$ be the set of all infinite sequences in $Y$. A binary relation $\rightarrow$ between $S$ and $Y$ is called a convergence on $Y$ if it satisfies the following axioms:

(C1) If $x_{n} \rightarrow x$ and $y_{n} \rightarrow y$, then $x_{n}+y_{n} \rightarrow x+y$. 
(C2) If $x_{n} \rightarrow x$ and $\lambda \in \mathbb{R}$, then $\lambda x_{n} \rightarrow \lambda x$.

(C3) If $\lambda_{n} \rightarrow \lambda$ in $\mathbb{R}$ and $x \in Y$, then $\lambda_{n} x \rightarrow \lambda x$.

A real vector space $Y$ endowed with convergence is said to be a vector space with convergence. If $x_{n} \rightarrow x$, then $\left(x_{n}\right)$ is said to be a convergent sequence in $Y$, and the vector $x$ is said to be a limit of $\left(x_{n}\right)$.

Definition $2.2([4])$ Let $(Y, \rightarrow)$ be a vector space with convergence. An ordering $\preceq$ on $Y$ is said to be a vector ordering if it is compatible with the algebraic and convergence structures on $Y$ in the sense that the following are true:

(V1) If $x \preceq y$, then $x+z \preceq y+z$.

(V2) If $\lambda \geq 0$ and $x \leq y$, then $\lambda x \preceq \lambda y$.

(V3) If $x_{n} \rightarrow x, y_{n} \rightarrow y, x_{n} \preceq y_{n}$ for all $n$, then $x \preceq y$.

A vector space with convergence $Y$ endowed with vector ordering is called an ordered vector space with convergence.

Definition 2.3 ([4]) Let $(Y, \preceq, \rightarrow)$ be an ordered vector space with convergence. A strict ordering $\prec$ on $Y$ is said to be a strict vector ordering if it is compatible with the vector ordering, the algebraic structure and the convergence structure on $Y$ in the sense that the following are true:

(S1) If $x \prec y$, then $x \preceq y$.

(S2) If $x \preceq y$ and $y \prec z$, then $x \prec z$.

(S3) If $x \prec y$, then $x+z \prec y+z$.

(S4) If $\lambda>0$ and $x \prec y$, then $\lambda x \prec \lambda y$.

(S5) If $x_{n} \rightarrow x, y_{n} \rightarrow y$ and $x \prec y$, then $x_{n} \prec y_{n}$ for all but finitely many $n$.

It turns out that an ordered vector space can be endowed with at most one strict vector ordering (see Proinov [4, Theorem 5.1]).

Definition 2.4 (Solid vector space) An ordered vector space with convergence endowed with a strict vector ordering is said to be a solid vector space.

Let us consider an important example of a solid vector space.

Example 2.5 Let $(Y, \tau)$ be a topological vector space and $K \subset Y$ be a cone with nonempty interior $K^{\circ}$. Define the vector ordering $\preceq$ on $Y$ and the strict vector ordering $\prec$ on $Y$, respectively, by means of

$$
\begin{aligned}
& x \preceq y \quad \text { if and only if } \quad y-x \in K, \\
& x \prec y \text { if and only if } \quad y-x \in K^{\circ} .
\end{aligned}
$$

Then $Y$ is a solid vector space called a solid topological vector space.

Now let us recall the definition of a cone metric space known also as ' $K$-metric spaces' (see Zabrejko [2], Proinov [4] and references therein).

Definition 2.6 (Cone metric space) Let $X$ be a nonempty set, and let $(Y, \preceq)$ be an ordered vector space with convergence. A vector-valued function $d: X \times X \rightarrow Y$ is said to be a cone metric on $Y$ if the following conditions hold: 
(i) $d(x, y) \succeq 0$ for all $x, y \in X$ and $d(x, y)=0$ if and only if $x=y$;

(ii) $d(x, y)=d(y, x)$ for all $x, y \in X$;

(iii) $d(x, y) \preceq d(x, z)+d(z, y)$ for all $x, y, z \in X$.

The pair $(X, d)$ is called a cone metric space over $Y$.

Let $(X, d)$ be a cone metric space over a solid vector space $(Y, \preceq, \prec), x_{0} \in X$ and $r \in Y$ with $r \succ 0$. Then the set $U\left(x_{0}, r\right)=\left\{x \in X: d\left(x, x_{0}\right) \prec r\right\}$ is called an open ball with center $x_{0}$ and radius $r$.

Every cone metric space $X$ over a solid vector space $Y$ is a Hausdorff topological space with topology generated by the basis of all open balls. Then a sequence $\left(x_{n}\right)$ of points in $X$ converges to $x \in X$ if and only if for every vector $c \in Y$ with $c \succ 0, d\left(x_{n}, x\right) \prec c$ for all but finitely many $n$.

Recall also that a sequence $\left(x_{n}\right)$ in $X$ is called a Cauchy sequence if for every $c \in Y$ with $c \succ 0$ there is $N \in \mathbb{N}$ such that $d\left(x_{n}, x_{m}\right) \prec c$ for all $n, m>N$. A cone metric space $X$ is called complete if each Cauchy sequence in $X$ is convergent.

In order to prove our main result we need the following two theorems.

Theorem $2.7([4])$ Let $(X, d)$ be a complete cone metric space over a solid vector space $(Y, \preceq)$. Suppose $\left(x_{n}\right)$ is a sequence in $X$ satisfying

$$
d\left(x_{n}, x_{m}\right) \preceq b_{n} \text { for all } n, m \geq 0 \text { with } m \geq n,
$$

where $\left(b_{n}\right)$ is a sequence in $Y$ which converges to 0 . Then $\left(x_{n}\right)$ converges to a point $\xi \in X$ with error estimate

$$
d\left(x_{n}, \xi\right) \preceq b_{n} \quad \text { for all } n \geq 0 .
$$

Theorem $2.8([4])$ Let $(X, d)$ be a cone metric space over a solid vector space $(Y, \preceq)$ and $T: X \rightarrow X$. Suppose that for some $x \in X$, the Picard iteration $\left(T^{n} x\right)$ converges to a point $\xi \in X$. Suppose also that there exist nonnegative numbers $\alpha$ and $\beta$ such that

$$
d(\xi, T \xi) \preceq \alpha d(x, \xi)+\beta d(T x, \xi) \quad \text { for each } x \in X
$$

Then $\xi$ is a fixed point of $T$.

\section{Auxiliary results}

Let $A$ be a subset of a real vector space $Y$. Recall that the convex hull of $A$, denoted $\operatorname{co} A$, is the smallest convex set including $A$. Suppose $x, x_{1}, \ldots, x_{n} \in Y$. It is well known that $x \in$ $\operatorname{co}\left\{x_{1}, \ldots, x_{n}\right\}$ if and only if there exist nonnegative numbers $\alpha_{1}, \ldots, \alpha_{n}$ such that $\sum_{i=1}^{n} \alpha_{i}=1$ and $x=\sum_{i=1}^{n} \alpha_{i} x_{i}$.

Lemma 3.1 Let $(Y, \preceq)$ be an ordered vector space. Suppose that $x, y, x_{1}, \ldots, x_{n}, y_{1}, \ldots, y_{m}$ are vectors in $Y$ and $\lambda$ is a real number. Then:

(P1) $x \preceq \operatorname{co}\left\{x_{1}, \ldots, x_{n}\right\} \Rightarrow x \preceq \operatorname{co}\left\{x_{1}, \ldots, x_{n}, y\right\}$;

(P2) $x \preceq \operatorname{co}\left\{x_{1}, \ldots, x_{n}\right\}$ and $x_{i} \preceq y_{i}$ for all $i \Rightarrow x \preceq \operatorname{co}\left\{y_{1}, \ldots, y_{n}\right\}$;

(P3) $x \preceq \operatorname{co}\left\{x_{1}, \ldots, x_{n}, y\right\}$ and $y \preceq \operatorname{co}\left\{y_{1}, \ldots, y_{m}\right\} \Rightarrow x \preceq \operatorname{co}\left\{x_{1}, \ldots, x_{n}, y_{1}, \ldots, y_{m}\right\}$;

(P4) $x \preceq \operatorname{co}\left\{0, x_{1}, \ldots, x_{n}\right\} \Leftrightarrow x \preceq \operatorname{co}\left\{x_{1}, \ldots, x_{n}\right\}$ if $x_{i} \succeq 0$ for some $i$; 
(P5) $x \preceq \operatorname{co}\left\{\lambda x, x_{1}, \ldots, x_{n}\right\} \Leftrightarrow x \preceq \operatorname{co}\left\{x_{1}, \ldots, x_{n}\right\}$ if $\lambda<1$ and $x_{i} \succeq 0$ for some $i$;

(P6) $x \preceq \operatorname{co}\left\{x_{1}, \ldots, x_{n}, y\right\} \Leftrightarrow x \preceq \operatorname{co}\left\{x_{1}, \ldots, x_{n}\right\}$ if $y=x_{i}$ for some $i$.

Proof We only prove the necessity of (P5) since the proofs of the other properties are similar. The inequality $x \preceq \operatorname{co}\left\{\lambda x, x_{1}, \ldots, x_{n},\right\}$ implies that there exist nonnegative numbers $\alpha, \alpha_{1}, \ldots, \alpha_{n}$ such that $\alpha+\sum_{i=1}^{n} \alpha_{i}=1$ and $x \preceq \alpha \lambda x+\sum_{i=1}^{n} \alpha_{i} x_{i}$. From this inequality and $\alpha \lambda<1$, we deduce

$$
x \preceq \sum_{i=1}^{n} \beta_{i} x_{i},
$$

where $\beta_{i}=\alpha_{i} /(1-\alpha \lambda)$. We have $\sum_{i=1}^{n} \beta_{i}=(1-\alpha) /(1-\alpha \gamma)<1$. By the assumptions, we have $x_{i} \succeq 0$ for some $i$. Without loss of generality we may assume that $x_{1} \succeq 0$. Define the nonnegative numbers $\gamma_{1}, \ldots, \gamma_{n}$ by $\gamma_{1}=1-\sum_{j=2}^{n} \beta_{j}$ and $\gamma_{i}=\beta_{i}$ for $i \geq 2$. From (6) and $\beta_{1} \leq \gamma_{1}$, we obtain

$$
x \preceq \sum_{i=1}^{n} \gamma_{i} x_{i} .
$$

This implies $x \preceq \operatorname{co}\left\{x_{1}, \ldots, x_{n}\right\}$ since $\sum_{i=1}^{n} \gamma_{i}=1$.

Remark 3.2 Note that Lemma 3.1 remains true if we omit the expression 'co' from its formulation.

The following lemma was given by Zhang [19, Lemma 6] in a slightly different form. We give a simple proof of this lemma.

Lemma 3.3 Let $(X, d)$ be a cone metric space over an ordered vector space $(Y, \preceq)$, $T: X \rightarrow X$ be a quasi-contraction with contraction constant $\lambda \in[0,1)$, and let $x \in X$. Then for every $m \in \mathbb{N}$, we have

$$
d\left(T^{i} x, T^{m} x\right) \preceq \lambda^{i} \operatorname{co}\left\{d(x, T x), \ldots, d\left(x, T^{m} x\right)\right\} \quad \text { for } i=1, \ldots, m
$$

Proof We prove the statement by induction on $m$. It is obviously true for $m=1$. Assume that $n \in \mathbb{N}$ and assume that (7) is satisfied for any natural number $m \leq n$. We have to prove that

$$
d\left(T^{i} x, T^{n+1} x\right) \preceq \lambda^{i} \operatorname{co}\left\{d(x, T x), \ldots, d\left(x, T^{n+1} x\right)\right\} \quad \text { for } i=1, \ldots, n+1
$$

We divide the proof of (8) into three steps.

Step 1 . We claim that for every natural number $i \leq n$ the following inequality holds:

$$
d\left(T^{i} x, T^{n+1} x\right) \preceq \operatorname{co}\left\{\lambda^{i} d(x, T x), \ldots, \lambda^{i} d\left(x, T^{n} x\right), \lambda d\left(T^{n} x, T^{n+1} x\right), \lambda d\left(T^{i-1} x, T^{n+1} x\right)\right\} .
$$


By the definition of the quasi-contraction mapping, we obtain

$$
\begin{aligned}
& d\left(T^{i} x, T^{n+1} x\right) \\
& \quad=d\left(T\left(T^{i-1} x\right), T\left(T^{n} x\right)\right) \\
& \quad \preceq \lambda \operatorname{co}\left\{d\left(T^{i-1} x, T^{n} x\right), d\left(T^{i-1} x, T^{i} x\right), d\left(T^{n} x, T^{n+1} x\right), d\left(T^{i-1} x, T^{n+1} x\right), d\left(T^{i} x, T^{n} x\right)\right\} .
\end{aligned}
$$

From the induction hypothesis and properties (P1) and (P2), we get the following three inequalities:

$$
\begin{aligned}
& d\left(T^{i-1} x, T^{n} x\right) \preceq \lambda^{i-1} \operatorname{co}\left\{d(x, T x), \ldots, d\left(x, T^{n} x\right)\right\}, \\
& d\left(T^{i-1} x, T^{i} x\right) \preceq \lambda^{i-1} \operatorname{co}\left\{d(x, T x), \ldots, d\left(x, T^{n} x\right)\right\}, \\
& d\left(T^{i} x, T^{n} x\right) \preceq \lambda^{i-1} \operatorname{co}\left\{d(x, T x), \ldots, d\left(x, T^{n} x\right)\right\} .
\end{aligned}
$$

From the last four inequalities and properties (P3) and (P6), we obtain the desired inequality.

Step 2. We claim that for every natural number $i \leq n$ the following inequality holds:

$$
d\left(T^{i} x, T^{n+1} x\right) \preceq \operatorname{co}\left\{\lambda^{i} d(x, T x), \ldots, \lambda^{i} d\left(x, T^{n+1} x\right), \lambda d\left(T^{n} x, T^{n+1} x\right)\right\}
$$

We prove this by finite induction on $i$. Setting $i=1$ in the claim of Step 1 , we immediately arrive at the following inequality:

$$
d\left(T x, T^{n+1} x\right) \preceq \operatorname{co}\left\{\lambda d(x, T x), \ldots, \lambda d\left(x, T^{n} x\right), \lambda d\left(T^{n} x, T^{n+1} x\right), \lambda d\left(x, T^{n+1} x\right)\right\},
$$

which proves the claim of Step 2 for $i=1$. Assume that for some $i \leq n$, the claim of Step 2 holds. Now we shall show that

$$
d\left(T^{i+1} x, T^{n+1} x\right) \preceq \operatorname{co}\left\{\lambda^{i+1} d(x, T x), \ldots, \lambda^{i+1} d\left(x, T^{n+1} x\right), \lambda d\left(T^{n} x, T^{n+1} x\right)\right\} .
$$

It follows from Step 1 that

$$
\begin{aligned}
& d\left(T^{i+1} x, T^{n+1} x\right) \\
& \quad \preceq \operatorname{co}\left\{\lambda^{i+1} d(x, T x), \ldots, \lambda^{i+1} d\left(x, T^{n+1} x\right), \lambda d\left(T^{n} x, T^{n+1} x\right), \lambda d\left(T^{i} x, T^{n+1} x\right)\right\} .
\end{aligned}
$$

By the finite induction hypothesis and property (P2), we have

$$
d\left(T^{i} x, T^{n+1} x\right) \preceq \operatorname{co}\left\{\lambda^{i} d(x, T x), \ldots, \lambda^{i} d\left(x, T^{n+1} x\right), d\left(T^{n} x, T^{n+1} x\right)\right\}
$$

From (10), (11), and properties (P3) and (P6), we obtain (9).

Step 3 . Now we shall prove (8). From the claim of Step 2 with $i=n$, we get

$$
d\left(T^{n} x, T^{n+1} x\right) \preceq \operatorname{co}\left\{\lambda^{n} d(x, T x), \ldots, \lambda^{n} d\left(x, T^{n+1} x\right), \lambda d\left(T^{n} x, T^{n+1} x\right)\right\}
$$

According to the property (P5), this inequality is equivalent to

$$
d\left(T^{n} x, T^{n+1} x\right) \preceq \lambda^{n} \operatorname{co}\left\{d(x, T x), \ldots, d\left(x, T^{n+1} x\right)\right\},
$$


which by (P2) implies

$$
d\left(T^{n} x, T^{n+1} x\right) \preceq \lambda^{i-1} \operatorname{co}\left\{d(x, T x), \ldots, d\left(x, T^{n+1} x\right)\right\} .
$$

Finally, by the claim of Step 2 and the inequality (12), taking into account the properties (P3) and (P6), we obtain (8). This completes the proof of the lemma.

In the following lemma, we show that if $T$ is a quasi-contraction of a cone metric space $X$, then for every starting point $x \in X$, the Picard iteration sequence $\left(T^{n} x\right)$ is bounded in the space $X$.

Lemma 3.4 Let $(X, d)$ be a cone metric space over an ordered vector space $(Y, \preceq)$, $T: X \rightarrow X$ be a quasi-contraction with contraction constant $\lambda \in[0,1)$, and let $x \in X$. Then for every $m \in \mathbb{N}$, we have

$$
d\left(x, T^{m} x\right) \preceq \frac{1}{1-\lambda} d(x, T x) .
$$

Proof We prove the statement by induction on $m$. If $m=1$, then inequality (13) holds since $0 \leq \lambda<1$. Assume that $n \in \mathbb{N}$ and assume that (7) is satisfied for any natural number $m \leq n$. Then we have to prove that

$$
d\left(x, T^{n+1} x\right) \preceq \frac{1}{1-\lambda} d(x, T x) .
$$

From the triangle inequality, we obtain

$$
d\left(x, T^{n+1} x\right) \preceq d(x, T x)+d\left(T x, T^{n+1} x\right) .
$$

By Lemma 3.3, we get

$$
d\left(T x, T^{n+1} x\right) \preceq \lambda \operatorname{co}\left\{d(x, T x), \ldots, d\left(x, T^{n+1} x\right)\right\} .
$$

By the induction hypothesis, we have that (13) holds for all $m \leq n$. Then it follows from (16), (P2), and (P6) that

$$
d\left(T x, T^{n+1} x\right) \preceq \operatorname{co}\left\{\frac{\lambda}{1-\lambda} d(x, T x), \lambda d\left(x, T^{n+1} x\right)\right\} .
$$

This inequality implies that there exists $\alpha \in[0,1]$ such that

$$
d\left(T x, T^{n+1} x\right) \preceq \alpha \frac{\lambda}{1-\lambda} d(x, T x)+(1-\alpha) \lambda d\left(x, T^{n+1} x\right) .
$$

Combining (15) and (17), we get

$$
d\left(x, T^{n+1} x\right) \preceq d(x, T x)+\alpha \frac{\lambda}{1-\lambda} d(x, T x)+(1-\alpha) \lambda d\left(x, T^{n+1} x\right),
$$

which is equivalent to the following inequality:

$$
(1-\lambda+\alpha \lambda) d\left(x, T^{n+1} x\right) \preceq \frac{1-\lambda+\alpha \lambda}{1-\lambda} d(x, T x) .
$$


Multiplying both sides of this inequality by $1 /(1-\lambda+\alpha \lambda)$, we obtain (14). This completes the proof of the lemma.

Lemma 3.5 Let $(X, d)$ be a cone metric space over an ordered vector space $(Y, \preceq)$, and let $T: X \rightarrow X$ be a quasi-contraction with contraction constant $\lambda \in[0,1)$. Then for all $x, y \in X$, we have

$$
d(x, T x) \preceq \alpha d(x, y)+\beta d(x, T y)
$$

where $\alpha=\lambda /(1-\lambda)$ and $\beta=(1+\lambda) /(1-\lambda)$.

Proof Let $x, y \in X$ be fixed. First we shall prove that

$$
d(T x, T y) \preceq \lambda(d(x, y)+d(x, T x)+d(x, T y)) .
$$

It follows from Definition 1.5 that there exist five nonnegative numbers $\alpha, \beta, \gamma, \mu, \nu$ such that $\alpha+\beta+\gamma+\mu+v=1$ and

$$
d(T x, T y) \preceq \lambda(\alpha d(x, y)+\beta d(x, T x)+\gamma d(y, T y)+\mu d(x, T y)+v d(y, T x)) .
$$

From this and the inequalities

$$
d(y, T y) \preceq d(x, y)+d(x, T y) \quad \text { and } \quad d(y, T x) \preceq d(x, y)+d(x, T x)
$$

we obtain

$$
d(T x, T y) \preceq \lambda((\alpha+\gamma+v) d(x, y)+(\beta+\nu) d(x, T x)+(\gamma+\mu) d(x, T y)) .
$$

This inequality yields (19) since $\alpha+\gamma+\nu \leq 1, \beta+\nu \leq 1$ and $\gamma+\mu \leq 1$. Now we are ready to prove (18). From the triangle inequality, we get

$$
d(x, T x) \preceq d(x, T y)+d(T x, T y) .
$$

From this and (19), we obtain

$$
d(x, T x) \preceq d(x, T y)+\lambda(d(x, y)+d(x, T x)+d(x, T y)),
$$

which can be presented in the following equivalent form:

$$
(1-\lambda) d(x, T x) \preceq \lambda d(x, y)+(1+\lambda) d(x, T y) .
$$

Multiplying both sides of this inequality by $1 /(1-\lambda)$, we get (18).

Lemma 3.6 Let $(X, d)$ be a cone metric space over an ordered vector space $(Y, \preceq)$. Then every quasi-contraction $T: X \rightarrow X$ has at most one fixed point in $X$.

Proof Suppose that $x$ and $y$ are two fixed points of $T$. It follows from the inequality (4) and properties (P4) and (P6) that $d(x, y) \preceq \lambda d(x, y)$ which implies $d(x, y) \preceq 0$. On the other hand, $d(x, y) \succeq 0$. Hence, $d(x, y)=0$, which yields $x=y$. 


\section{Main result}

Now we are ready to state the main result of this paper. Let $(X, d)$ be a complete cone metric space over an ordered vector space $Y$. Recall that for a point $x_{0} \in X$ and a vector $r \in Y$ with $r \succeq 0$, the set $\bar{U}\left(x_{0}, r\right)=\left\{x \in X: d\left(x, x_{0}\right) \preceq r\right\}$ is called a closed ball with center $x_{0}$ and radius $r$.

Theorem 4.1 Let $(X, d)$ be a complete cone metric space over a solid vector space $(Y, \preceq)$, and let $T: X \rightarrow X$ be a quasi-contraction with contraction constant $\lambda \in[0,1)$. Then the following statements hold true:

(i) EXISTENCE, UNIQUENESS AND LOCALIZATION. T has a unique fixed point $\xi$ which belongs to the closed ball $\bar{U}(x, r)$ with radius

$$
r=\frac{1}{1-\lambda} d(x, T x)
$$

where $x$ is any point in $X$.

(ii) Convergence of Picard iteration. Starting from any point $x \in X$ the Picard sequence $\left(T^{n} x\right)$ remains in the closed ball $\bar{U}(x, r)$ and converges to $\xi$.

(iii) A priori error estimate. For every point $x \in X$ the following a priori estimate holds:

$$
d\left(T^{n} x, \xi\right) \preceq \frac{\lambda^{n}}{1-\lambda} d(x, T x) \quad \text { for all } n \geq 0
$$

(iv) A posteriori error estimates. For every point $x \in X$ the following a posteriori estimate holds:

$$
\begin{array}{ll}
d\left(T^{n} x, \xi\right) \preceq \frac{1}{1-\lambda} d\left(T^{n} x, T^{n+1} x\right) & \text { for all } n \geq 0, \\
d\left(T^{n} x, \xi\right) \preceq \frac{\lambda}{1-\lambda} d\left(T^{n} x, T^{n-1} x\right) & \text { for all } n \geq 1 .
\end{array}
$$

Proof Let $x$ be an arbitrary point in $X$. By Lemma 3.3, for all $m, n \in \mathbb{N}$ with $m \geq n$, we have

$$
d\left(T^{n} x, T^{m} x\right) \preceq \lambda^{n} \operatorname{co}\left\{d(x, T x), \ldots, d\left(x, T^{m} x\right)\right\}
$$

From this, Lemma 3.4, and properties (P2) and (P6), we deduce

$$
d\left(T^{n} x, T^{m} x\right) \preceq b_{n}, \quad \text { where } b_{n}=\frac{\lambda^{n}}{1-\lambda} d(x, T x) .
$$

Note that $\left(b_{n}\right)$ is a sequence in $Y$ which converges to 0 since $\lambda^{n} \rightarrow 0$ in $\mathbb{R}$. Now applying Theorem 2.7 to the Picard sequence $\left(T^{n} x\right)$, we conclude that there exists a point $\xi \in X$ such that $\left(T^{n} x\right)$ converges to $\xi$ and $d\left(T^{n} x, \xi\right) \preceq b_{n}$ for every $n \geq 0$. The last inequality coincides with the estimate (20). Setting $n=0$ in (20), we get

$$
d(x, \xi) \preceq \frac{1}{1-\lambda} d(x, T x)
$$


which means that $\xi \in \bar{U}(x, r)$. The inequality (24) holds for every point $x \in X$. Applying (24) to the point $T^{n} x$, we obtain (21). Setting $n=1$ in (20), we get

$$
d(T x, \xi) \preceq \frac{\lambda}{1-\lambda} d(x, T x) .
$$

Applying (25) to the point $T^{n-1} x$, we get (22). Setting $n=0$ in (23), we obtain $d\left(x, T^{m} x\right) \preceq b_{0}$ for every $m \geq 0$. Hence, the sequence $\left(T^{n} x\right)$ lies in the ball $\bar{U}(x, r)$ since $r=b_{0}$.

It follows from Lemma 3.5 that $\xi$ satisfies condition (5). Hence, by Theorem 2.8, we conclude that $\xi$ is a fixed point of $T$. The uniqueness of the fixed point follows from Lemma 3.6.

Theorem 4.1 extends and complements the recent results of Ding et al. [24, Theorem 3.1] and Zhang [19, Theorem 3] as well as previous results due to Ilić and Rakočević [20, Theorem 2.1], Kadelburg et al. [21, Theorem 2.2], Rezapour et al. [23, Theorem 2.1] and Kadelburg et al. [8, Theorem 3.5(b)] who have studied quasi-contraction mappings of the type (3).

Theorem 4.1 also extends and complements the results of Abbas and Rhoades [39, Corollary 2.3], Olaleru [40, Theorem 2.1], Azam et al. [41, Theorem 2.2], Song et al. [42, Corollary 2.1]. These authors have studied the class of mappings satisfying a contractive condition of the type

$$
d(T x, T y) \preceq \alpha d(x, y)+\beta d(x, T x)+\gamma d(y, T y)+\mu d(x, T y)+\nu d(y, T x)
$$

for all $x, y \in X$, where $\alpha, \beta, \gamma, \mu$ and $v$ are five nonnegative constants such that $\alpha+\beta+\gamma+$ $\mu+v<1$. In this case Theorem 4.1 holds with $\lambda=\alpha+\beta+\gamma+\mu+v$ since condition (26) implies condition (4) with this $\lambda$. Let us note that in this special case Theorem 4.1 holds even with $\lambda=(\alpha+\delta) /(1-\delta)$, where $\delta=(\beta+\gamma+\mu+\nu) / 2$.

\section{Examples}

Zhang [19, Example 1] gives an example showing that the set of all quasi-contractions of the type (3) is a proper subset of the set of all quasi-contractions defined by (4). In order to prove this, he considers a selfmapping of a cone metric space $X$ over a normal solid vector space $Y$. Ding et al. [24, Example 4.1] provide a similar example, but for the case of a non-normal solid vector space $Y$.

The aim of this section is to unify these two examples. Let $\mathcal{B}$ denote the set of all quasicontractions of the type (3), and let $\mathcal{C}$ denote the set of all quasi-contractions defined by (4), that is,

$$
\begin{aligned}
& \mathcal{B}=\{T: X \rightarrow X \mid d(T x, T y) \preceq \lambda\{d(x, y), d(x, T x), d(y, T y), d(x, T y), d(y, T x)\}\}, \\
& \mathcal{C}=\{T: X \rightarrow X \mid d(T x, T y) \preceq \lambda \operatorname{co}\{d(x, y), d(x, T x), d(y, T y), d(x, T y), d(y, T x)\}\} .
\end{aligned}
$$

Now we shall construct a family of examples which show that $\mathcal{B}$ is a proper subset of $\mathcal{C}$. In particular, this family contains both the example of Zhang [19] and the example of Ding et al. [24]. 
Definition 5.1 Let $(Y, \preceq)$ be an ordered vector space, and let $a, b, c \succeq 0$ be three vectors in $Y$. We say that the triple $(a, b, c)$ satisfies property $(C)$ if the following two statements hold:

- $a \preceq \lambda \operatorname{co}\{b, c\}$ for some $\lambda \in[0,1), b \preceq a+c$ and $c \preceq a+b$.

- $a \preceq k\{b, c\}$ is wrong for every $k \in[0,1)$.

Proposition 5.2 Let $Y=\mathbb{R}$ be endowed with the usual ordering $\leq$. Then there are no triples $(a, b, c)$ in $Y$ satisfying property $(C)$.

Proof Assume that there is a triple $(a, b, c)$ in $Y$ with property (C). Then $a \preceq \lambda \max \{b, c\}$ for some $\lambda \in[0,1)$. On the other hand, $a \preceq k \max \{b, c\}$ is wrong for every $k \in[0,1)$. This is a contradiction which proves the proposition.

Proposition 5.3 Let $Y=\mathbb{R}^{n}(n \geq 2)$ be endowed with coordinate-wise ordering $\preceq$. Then in $Y$ there exist infinitely many triples $(a, b, c)$ satisfying property $(C)$.

Proof Choose three real numbers $\alpha, \beta$ and $\gamma$ such that

$$
\beta<\gamma<3 \beta \quad \text { and } \quad \max \{\beta, \gamma-\beta\} \leq \alpha<\frac{\beta+\gamma}{2} .
$$

Then the vectors $a=(\alpha, \ldots, \alpha), b=(\beta, \ldots, \beta, \gamma)$ and $c=(\gamma, \ldots, \gamma, \beta)$ satisfy property $(C)$ with $\lambda=\frac{2 \alpha}{\beta+\gamma}$.

Proposition 5.4 Let $Y=C^{n}[0,1](n \geq 2)$ be endowed with point-wise ordering $\preceq$. Then in $Y$ there exist infinitely many triples $(a, b, c)$ satisfying property $(C)$.

Proof Choose three real numbers $\alpha, \beta$ and $\gamma$ as in the proof of Proposition 5.3, then choose a real number $\delta$ such that

$$
\gamma-\alpha \leq \delta<\gamma \quad \text { and } \quad \delta \leq \frac{\alpha+\gamma-\beta}{2} .
$$

Then the functions $a(t)=\alpha, b(t)=\beta+\delta t$ and $c(t)=\gamma-\delta t$ satisfy property (C) with $\lambda=$ $\frac{2 \alpha}{\beta+\gamma}$.

Example 5.5 Let $(Y, \preceq)$ be an arbitrary solid vector space, and let $(a, b, c)$ be any triple in $Y$ with property (C). Furthermore, let $X=\{x, y, z\}$, and let $d: X \times X \rightarrow Y$ be defined by

$$
d(x, y)=a, \quad d(x, z)=b, \quad d(y, z)=c,
$$

$d(u, v)=d(u, v)$ and $d(u, u)=0$ for $u, v \in X$. Then $(X, d)$ is a complete cone metric space over $Y$ since the triple $(a, b, c)$ satisfies property $(C)$. Consider now the mapping $T: X \rightarrow X$ defined by

$$
T x=x, \quad T y=x, \quad T z=y .
$$

Using Lemma 3.1, it is easy to prove that $T \in \mathcal{C}$ if and only if $a \preceq \lambda \operatorname{co}\{b, c\}$ for some $\lambda \in$ $[0,1)$. Analogously, taking into account Remark 3.2 , one can easily prove that $T \in \mathcal{B}$ if 
and only if $a \preceq k\{b, c\}$ for some $k \in[0,1)$. Now taking into account that the triple $(a, b, c)$ satisfies property $(\mathrm{C})$, we conclude that $T \in \mathcal{C}$ and $T \notin \mathcal{B}$. Hence, $\mathcal{B}$ is a proper subset of $\mathcal{C}$.

\section{Competing interests}

The authors declare that they have no competing interests.

\section{Authors' contributions}

Both authors contributed equally and significantly in writing this paper. Both authors read and approved the final manuscript.

\section{Acknowledgements}

The research is supported by Project N113 FMI-002 of Plovdiv University.

Received: 19 February 2014 Accepted: 23 May 2014 Published: 03 Jun 2014

\section{References}

1. Collatz, L: Functional Analysis and Numerical Mathematics. Academic Press, New York (1966)

2. Zabrejko, PP: K-Metric and K-normed linear spaces: survey. Collect. Math. 48, 825-859 (1997)

3. Janković, S, Kadelburg, Z, Radenović, S: On cone metric spaces: a survey. Nonlinear Anal. 74, 2591-2601 (2011)

4. Proinov, PD: A unified theory of cone metric spaces and its applications to the fixed point theory. Fixed Point Theory Appl. 2013, Article ID 103 (2013)

5. Du, W-S: A note on cone metric fixed point theory and its equivalence. Nonlinear Anal. 72, 2259-2261 (2010)

6. Amini-Harandi, A, Fakhar, M: Fixed point theory in cone metric spaces obtained via the scalarization method. Comput. Math. Appl. 59, 3529-3534 (2010)

7. Feng, Y, Mao, W: The equivalence of cone metric spaces and metric spaces. Fixed Point Theory 11, 259-263 (2010)

8. Kadelburg, Z, Radenović, S, Rakočević, V: A note on the equivalence of some metric and cone metric fixed point results. Appl. Math. Lett. 24, 370-374 (2011)

9. Asadi, M, Rhoades, BE, Soleimani, H: Some notes on the paper 'The equivalence of cone metric spaces and metric spaces.' Fixed Point Theory Appl. 2012, Article ID 87 (2012)

10. Ercan, Z: On the end of the cone metric spaces. Topol. Appl. 166, 10-14 (2014)

11. Banach, S: Sur les opérations dans les ensembles abstraits et leurs applications aux équations integrals. Fundam. Math. 3, 133-181 (2009)

12. Berinde, V: Iterative Approximation of Fixed Points. Lecture Notes in Mathematics, vol. 1912. Springer, Berlin (2007)

13. Radenović, S, Kadelburg, Z: Quasi-contractions on symmetric and cone symmetric spaces. Banach J. Math. Anal. 5, 38-50 (2011)

14. Ćirić, LB: A generalization of Banach's contraction principle. Proc. Am. Math. Soc. 45, 267-273 (1974)

15. Proinov, PD: A generalization of the Banach contraction principle with high order of convergence of successive approximations. Nonlinear Anal. 67, 2361-2369 (2007)

16. Proinov, PD: New general convergence theory for iterative processes and its applications to Newton-Kantorovich type theorems. J. Complex. 26, 3-42 (2010)

17. Zhang, X: Fixed point theorem of generalized operator quasi-contractive mapping in cone metric spaces. Afr. Math 25, $135-146$ (2014)

18. Ali, MU, Kamran, T, Karapinar, E: A new approach to $(\alpha, \psi)$-contractive nonself multivalued mappings. J. Inequal. Appl. 2014, Article ID 71 (2014)

19. Zhang, X: Fixed point theorem of generalized quasi-contractive mapping in cone metric space. Comput. Math. Appl. 62, 1627-1633 (2011)

20. Ilić, D, Rakočević, V: Quasi-contraction on a cone metric space. Appl. Math. Lett. 22, $728-731$ (2009)

21. Kadelburg, Z, Radenović, S, Rakočević, V: Remarks on 'Quasi-contraction on a cone metric space'. Appl. Math. Lett. 22, 1674-1679 (2009)

22. Gajić, L, Rakočević, V: Quasi-contractions on a nonnormal cone metric spaces. Funct. Anal. Appl. 46, 62-65 (2012)

23. Rezapour, S, Haghi, RH, Shahzad, N: Some notes on fixed points of quasi-contraction maps. Appl. Math. Lett. 23, 498-502 (2010)

24. Ding, H-S, Jovanović, M, Kadelburg, Z, Radenović, S: Common fixed point results for generalized quasicontractions in tvs-cone metric spaces. J. Comput. Anal. Appl. 15, 463-470 (2013)

25. Karapinar, E: Fixed point theorems in cone Banach spaces. Fixed Point Theory Appl. 2009, Article ID 609281 (2009)

26. Karapinar, E: Some nonunique fixed point theorems of Ćirić type on cone metric spaces. Abstr. Appl. Anal. 2010 Article ID 123094 (2010)

27. Karapinar, E: Couple fixed point theorems for nonlinear contractions in cone metric spaces. Comput. Math. Appl. 59, 3656-3668 (2010)

28. Khamsi, MA: Remarks on cone metric spaces and fixed point theorems of contractive mappings. Fixed Point Theory Appl. 2010, Article ID 315398 (2010)

29. Dukić, D, Paunović, L, Radenović, S: Convergence of iterates with errors of uniformly quasi-Lipschitzian mappings in cone metric spaces. Kragujev. J. Math. 35, 399-410 (2011)

30. Karapinar, E, Kumam, P, Sintunavarat, W: Coupled fixed point theorems in cone metric spaces with a $c$-distance and applications. Fixed Point Theory Appl. 2012, Article ID 194 (2012)

31. Radenović, S: A pair of non-self mappings in cone metric spaces. Kragujev. J. Math. 36, 189-198 (2012)

32. Aydi, H, Karapinar, E, Mustafa, Z: Coupled coincidence point results on generalized distance in ordered cone metric spaces. Positivity 17, 979-993 (2013) 
33. Du, W-S, Karapinar, E: A note on cone $b$-metric and its related results: generalizations or equivalence? Fixed Point Theory Appl. 2013, Article ID 210 (2013)

34. Kadelburg, Z, Radenović, S: A note on various types of cones and fixed point results in cone metric spaces. Asian J. Math. Appl. 2013, 0104 (2013)

35. Popović, B, Radenović, S, Shukla, S: Fixed point results to tvs-cone b-metric spaces. Gulf J. Math. 1, 51-64 (2013)

36. Xu, S, Radenović, S: Fixed point theorems of generalized Lipschitz mappings on cone metric spaces over Banach algebra without assumption of normality. Fixed Point Theory Appl. 2014, Article ID 101 (2014)

37. Proinov, PD, Cholakov, SI: Semilocal convergence of Chebyshev-like root-finding method for simultaneous approximation of polynomial zeros. Appl. Math. Comput. 236, 669-682 (2014)

38. Proinov, PD, Cholakov, SI: Convergence of Chebyshev-like method for simultaneous computation of multiple polynomial zeros. C. R. Acad. Bulg. Sci. 67 (2014, in press)

39. Abbas, M, Rhoades, BE: Fixed and periodic point results in cone metric spaces. Appl. Math. Lett. 22, 511-515 (2009)

40. Olaleru, JO: Some generalizations of fixed point theorems in cone metric spaces. Fixed Point Theory Appl. 2009, Article ID 657914 (2009)

41. Azam, A, Beg, I, Arshad, M: Fixed point in topological vector space-valued cone metric spaces. Fixed Point Theory Appl. 2010, Article ID 604084 (2010)

42. Song, G, Sun, X, Zhao, Y, Wang, G: New common fixed point theorems for maps on cone metric spaces. Appl. Math Lett. 23, 1033-1037 (2010)

10.1186/1029-242X-2014-226

Cite this article as: Proinov and Nikolova: Iterative approximation of fixed points of quasi-contraction mappings in cone metric spaces. Journal of Inequalities and Applications 2014, 2014:226

\section{Submit your manuscript to a SpringerOpen ${ }^{\circ}$ journal and benefit from:}

- Convenient online submission

- Rigorous peer review

- Immediate publication on acceptance

- Open access: articles freely available online

- High visibility within the field

- Retaining the copyright to your article 